\title{
TEMPERATURE DEPENDENCE OF FERROFLUID SUSCEPTIBILITY. INFLUENCE OF PARTICLE SIZE DISTRIBUTION
}

\author{
Yu.I.Dikansky ${ }^{1}$, A.G.Ispiryan ${ }^{1}$, S.A.Kunikin ${ }^{1}$, A.V.Rodionov ${ }^{2}$ \\ 1 North-Caucasus Federal University, 1 Pushkina str. 355009, Stavropol, Russia \\ ${ }^{2}$ Admiral Makarov National University of Shipbuilding, \\ 9 Heroes of Ukraine str., 54000, Nova Odesa, Mykolaivs'ka oblast, Ukraine
}

\begin{abstract}
Investigations of temperature dependences of dynamic susceptibility for three ferrofluid samples with different size distributions are presented. These studies were performed at different sampling frequencies under the action of an external DC magnetic field. Analysis was done in terms of a Debye-like theory of complex susceptibility. It was also found that the temperature corresponding to maximum of the imaginary component of the complex magnetic susceptibility depends on the frequency of the probing field as a Vogel-Fulcher-like law.
\end{abstract}

Introduction. The temperature dependence of the magnetic susceptibility of ferrofluids has been studied in a number of papers [1-5], where maxima of this dependence are reported. In some cases, these maxima, corresponding to the temperatures of the carrier solidification, can be explained in terms of the blocking of particle Brownian degrees of freedom [1,2]. In [3], it was concluded that such maxima are related to a superparamagnetic-ferromagnetic transition. Some researchers assume that the maxima are caused by the phase transition of an ensemble of magnetic particles to a super-spin glass state (also known as the dipolar glass state).

The aim of our study is to reveal origins of the extremum of the temperature dependence of the ferrofluid AC susceptibility and its influence of interparticle interactions.

1. Samples and setup. The magnetic susceptibility was measured with the help of a setup described in [6]. The AC susceptibility investigations were performed in a frequency range of $10 \mathrm{~Hz}$ to $10 \mathrm{kHz}$. The magnitude of the probing field was $20 \mathrm{~A} / \mathrm{m}$. Experiments were performed with ferrofluid samples consisting of magnetite particles suspended in kerosene. Oleic acid was used as a surfactant. The samples were synthesized at "Ferrohidrodinamica" (Ukraine) and differed in particles mean size (Table 1). The sample granulometry was varied with the help of a centrifuge and controlled by a SEM Hitachi SU8020 Series. The aggregative stability of the samples was characterized by optical microscopy.

2. Results and discussion. At first, we characterized the temperature dependence of the real component of the complex susceptibility: these results are shown in Fig. 1. It was found that the temperature dependences of the real component of magnetic susceptibility for all samples were not monotonic, reached their maxima, and each of them had certain peculiarities. For the case of samples

Table 1. Particle mean sizes.

\begin{tabular}{rccc}
\hline \hline Sample & no. 1 & no. 2 & no. 3 \\
Mean diameter $d,[\mathrm{~nm}]$ & 8.3 & 9.4 & 11.1 \\
\hline \hline
\end{tabular}




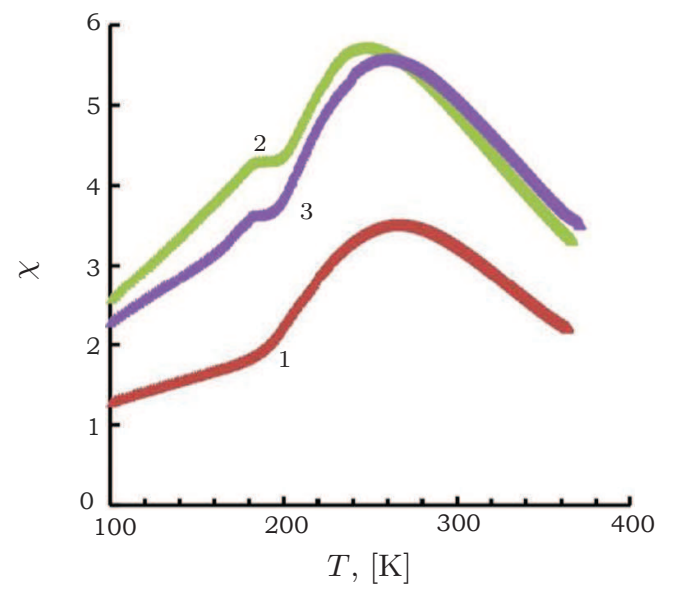

Fig. 1. Temperature dependence of the real component of ferrofluid dynamic magnetic susceptibility (the curve number corresponds to the sample number).

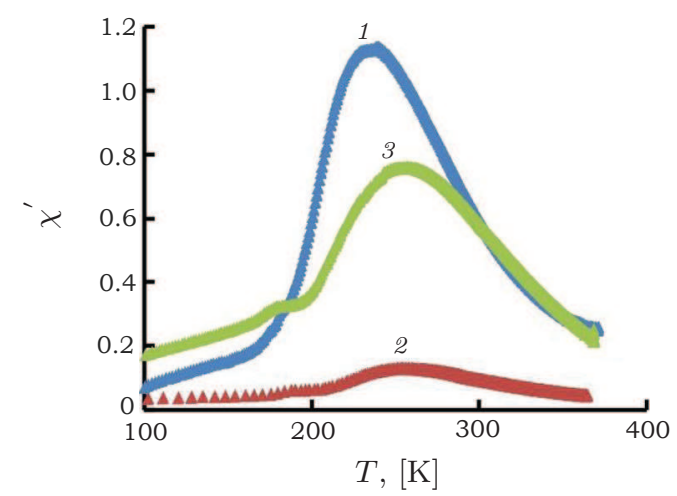

Fig. 2. Temperature dependence of the real part of dynamic magnetic susceptibility for sample 2 at different frequencies of the probing field: curve $1-320 \mathrm{~Hz} ; 2-2 \mathrm{kHz}$; $3-3 \mathrm{kHz}$.

2 and 3 , one can see a plateau stage in the region of carrier solidification and the sharp maxima in the room temperature region. Also it was found that the temperature corresponding to these maxima was frequency-dependent and shifted to the high temperature region, whereas the frequency increased (Fig. 2). The temperature dependence of the susceptibility imaginary component also reached a maximum, but at a smaller temperature than the real component (Fig. 3). Sample 3 was centrifuged for a longer time. As a result, the particle distribution function kurtosis is greater and the mean size is smaller than for samples 1 and 2 .

The temperature dependence of the real part of susceptibility had no plateau stage, whereas maxima at room temperature still existed.

Also it was found that the frequency behavior of the temperature maxima for sample 3 was the same as for other two samples. An additional DC magnetic field transformed the plateau to minima for samples 1 and 2 , as well as minima occurred for sample 3 in the same temperature region as for other samples. Also this additional field was the reason of shifting the room temperature maxima into the region of higher temperatures (Fig. 4). Furthermore, the temperature corresponding to the maxima increased with the DC magnetic field increase by about $5 \mathrm{kA} / \mathrm{m}$ up to its magnitude, but at higher magnitudes the temperature decreased. 
Temperature dependence of ferrofluid susceptibility. Influence of particle size...

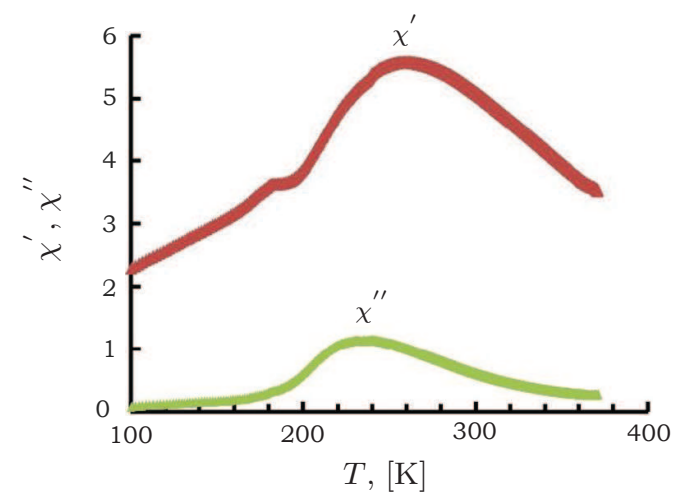

Fig. 3. Temperature dependence of the real and imaginary parts of dynamic magnetic susceptibility for sample 3 .

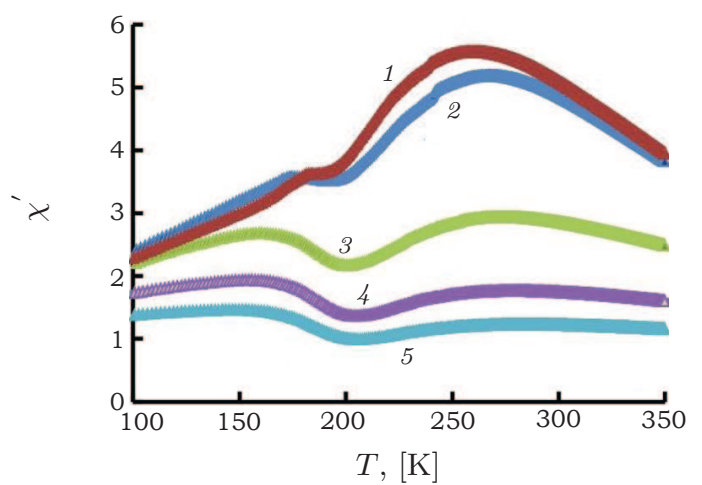

Fig. 4. Temperature dependence of the real part of dynamic magnetic susceptibility for sample 2 at different magnitudes of the external DC magnetic field: curve $1-0 \mathrm{kA} / \mathrm{m}$; 2- $0.86 \mathrm{kA} / \mathrm{m} ; 3-3.23 \mathrm{kA} / \mathrm{m} ; 4-6.45 \mathrm{kA} / \mathrm{m} ; 5-9.67 \mathrm{kA} / \mathrm{m}$.

It is possible to explain these minima in terms of the Brownian degrees of freedom de-blocking. Actually, while the sample was zero-field cooled, the magnetic moments of the particles were directed randomly. Besides, the external DC magnetic field is the cause of the particle magnetic moments orientation along the direction of the external magnetic field while the carrier was melting. The Brownian rotation made the oscillation magnitude decrease under the action of the probing AC magnetic field.

The plateau state at the temperature dependences of the real part of the $\mathrm{AC}$ susceptibility was caused first by the solid-liquid transition of the carrier. It can be also explained in terms of the enhancing interparticle interaction after a structure transformation in the ensemble of magnetic particles.

Earlier it was noted that the temperature corresponding to the maxima temperature dependences does not correspond to the melting point of the ferrofluid. One can assume [7] that the observed maxima of the temperature dependences of the real and imaginary parts of the $\mathrm{AC}$ susceptibility are caused by certain peculiarities of the relaxation processes in an ensemble of interacting Brownian magnetic particles. According to Debyes theory of complex AC susceptibility, the frequency dependence is given as

$$
\chi^{\prime}=\frac{\chi_{0}}{1+(\omega \tau)^{2}}
$$


and

$$
\chi^{\prime \prime}=\frac{\chi_{0} \omega \tau}{1+(\omega \tau)^{2}}
$$

where $\chi_{0}$ is the static susceptibility, $\omega$ is the frequency of the probing field, $\tau$ is the particle Brownian rotation time defined as

$$
\tau_{\mathrm{B}}=\frac{3 V \eta}{k T}
$$

with $V$ being the volume of a particle, $\eta$ is the carrier viscosity.

The prima facie to Eqs. (1),(2) assumes that they are not temperaturedependent. But it is known that both the static susceptibility and the relaxation time Eq. (3) are temperature-dependent. To compute the temperature dependences of $\chi^{\prime}$ and ", first, the temperature dependence of the static susceptibility and viscosity of the carrier (a $5 \%$ solution of oleic acid in kerosene) was investigated.

The numerical simulations of $\chi^{\prime}(T)$ and $\chi^{\prime \prime}(T)$ based on Eqs. (1),(2) and static investigations are illustrated in Fig. 5 . One can see that both values reach maxima but at different temperatures of the medium. It is no wonder that the maxima for were observed at lower temperatures than for $\chi^{\prime}(T)$. Fig. 5 presents a comparison of the experimental results and numerical simulations for $\chi^{\prime \prime}(T)$. It was found that a satisfactory agreement is possible only if in numerical simulations we use not the carrier viscosity, but the viscosity of ferrofluid. Besides, the temperatures corresponding to the maxima of $\chi^{\prime \prime}(T)$ in the experimental results and in the simulations are similar when the mean diameter of the particles used in the simulations is about $18 \mathrm{~nm}$, whereas the mean diameter determined by electronic microscopy is much smaller $(10 \mathrm{~nm})$. These differences are attributed to the interparticle interactions which increase the effective viscosity of the medium around the particle. From the analysis of the temperature dependence of the imaginary part of dynamic susceptibility it is possible to define the Brownian rotation time as a reverse frequency of the probing field at a temperature corresponding to the maxima of this dependence. Fig. 6 displays the results of its calculations for sample 1 (curve 1). In the same figure, curves 3 and 4 characterize the temperature dependence of the carrier and ferrofluid viscosity, respectively. It is well known that the temperature dependence of the fluid viscosity obeys the Arrhenius law as

$$
\eta=A \exp \left(\frac{W}{k T}\right) .
$$

From Eqs. (3) and (5) it can be expressed as

$$
\tau=\frac{3 V a}{k T} \exp \left(\frac{W}{k T}\right) .
$$

It is possible to find that the multiplier $1 / T$ is infinitesimally small with regards to $\exp (W / k T)$. The analysis allowed to conclude that the logarithms of the values $\tau$ and $\eta$ are directly proportional to $1 / T$ with the same rate of variation which is determined by the activation energy $W$.

In the figure we compare the experimental results for the relaxation time $\tau$ and viscosity $\eta$ at different temperatures of the medium with the dependence $\tau=B \exp (E / k T)$. One can see that this function gives a good approximation of the experimental data, however, its rates of variation are different. Hence, now we can conclude that the viscous activation energy $W_{\eta}$ and the activation energy of the magnetic moment orientation $W_{\tau}$ are not equal. This difference is caused by the influence of the interparticle interactions, which impedes the 
Temperature dependence of ferrofluid susceptibility. Influence of particle size...

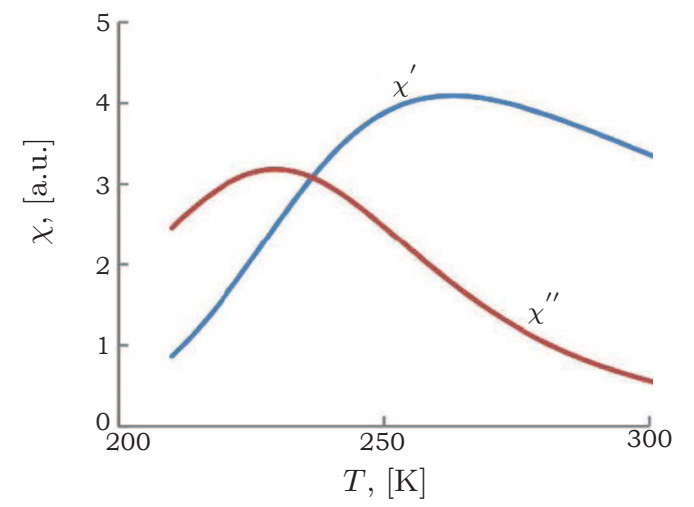

Fig. 5. Numerical simulations of the temperature dependence of the real and imaginary parts of dynamic susceptibility for sample 3.

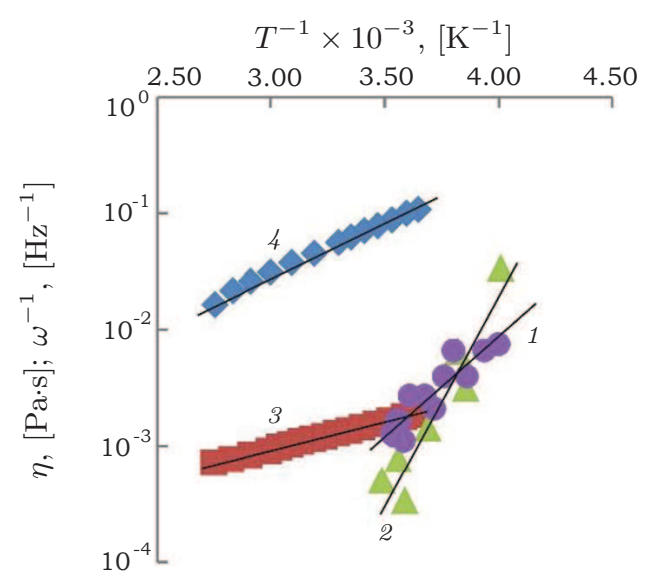

Fig. 6. Temperature dependence of the relaxation time of ferrofluid particles calculated by the temperature dependences of the imaginary part of magnetic susceptibility (1), by dispersion of magnetic susceptibility (2), by temperature dependences of the carrier viscosity (3) and viscosity of ferrofluid (4).

particle rotation by the acting probing field. The part of the energy corresponding to the interparticle interactions can be found as a difference between the activation energies, $\Delta W=W_{\eta}-W_{\tau}$. In our case, the value was about $\Delta W \approx 10 k T$ at room temperature.

Moreover, the activation energies determined from curves 1 and 3 are not equal either. This result can be explained by the increasing effective viscosity of the ferrofluid and relaxation time of the particles magnetic moments caused by the enhancing intensity of the interparticle interactions in the external magnetic field or by the chain aggregate formation. As a result, a shift of the $\chi(T)$ maxima to higher temperatures was observed. Now it is possible to conclude that the origin of the maxima for the $\chi(T)$ dependence is determined by the peculiarities of the Brownian rotation of magnetic particles.

This assumption was verified by investigating the frequency dependences of the AC susceptibility. The measurements were made in the range of temperatures corresponding to the maxima of the $\mathrm{AC}$ susceptibility temperature dependence. It 
was found that the imaginary part of the AC susceptibility monotonically increased while the sample was solid and while in the melting sample the temperature maxima were kept. These maxima correspond to the $90-150 \mathrm{~Hz}$ range of frequencies.

Note that the peak of the maximum of the frequency dependence of the imaginary part of dynamic susceptibility is temperature- and field-dependent. At low temperatures the maximum is sharper than at room temperature. When an additional magnetic field was imposed, the relative magnitude of the maximum increased and shifted to higher temperatures.

The investigation results on the dispersion of magnetic susceptibility made it possible to determine the relaxation time as a value proportional to a reverse frequency, which corresponds to the $\chi^{\prime \prime}$ maximum, and to find a dependence of this time on the temperature. These results are displayed in Fig. 6, curve 2. The found dependence satisfactory agrees with the same dependence determined from the susceptibility temperature dependences (cf. Fig. 6, curve 1). Some differences between the activation energies computed by curves 1 and 3 may be caused by the formation of aggregates.

But if consider the significant differences between the activation energies determined by the carrier and ferrofluid viscosities, by the relaxation time, by the large size of particles found in the simulations, we can conclude that in our case the used models are not accurate. Moreover, the increase of the maximum for $\chi^{\prime \prime}(T)$ in the additional magnetic field cannot be explained in terms of the relaxation of particles. One of the ways to solve this problem is to take into account the corresponding effects in the ensemble of magnetic particles.

Hypothetically, it might be caused by the occurring aggregates after melting of the carrier. Then aggregates dissolve with the temperature increase. It should be noted that, if compare curve 2 in Fig. 6 with the dependences of the carrier and ferrofluid viscosities on the reverse temperature, the activation energies of the particle rotation motion and translation motion differ significantly.

3. Conclusion. The obtained results allow to conclude that the temperature dependence of the AC susceptibility can vary in the range of temperatures corresponding to the carrier solidification, but in some cases, this variation does not produce maxima on this curve. In the case of a ferrofluid with relatively large particles, the plateau stage was observed. This plateau is caused by the interparticle interaction and by the emergence of particle aggregates after melting the carrier.

The transformation of the observed plateau to minimum under the external DC magnetic field is caused by the orientation of Brownian particles and aggregates along the magnetic field direction with the recovery of the Brownian degrees of freedom. The maximum of the temperature dependence of the AC susceptibility corresponding to the range of temperatures of the liquid carrier is determined by the particle magnetic moment relaxation processes. Furthermore, a strong interparticle interaction leads to the aggregation of the particles. The existence of aggregates is the reason of similar dependences of the relaxation processes for ferrofluids and for dipolar-glass systems.

Acknowledgements. We acknowledge financial support of the Russian Foundation for Basic Research (project no. 18-03-00279 A) and of the Ministry of Science and Education of the Russian Federation. We are also grateful to Prof. A. Aksenov (North-Caucasus Federal University) and to Prof. V. Avanikov (Ananikon Lab, Zelinsky Institute of Organic Chemistry, Moscow) for scanning electron microscopy. 
Temperature dependence of ferrofluid susceptibility. Influence of particle size...

\section{References}

[1] A.F. Pshenichnikov, A.V. Lebedev. Magnetic susceptibility of concentrated ferrocolloids. Colloid J., vol. 67 (2005), no. 2, pp. 189-200.

[2] A.F. Pshenichnikov, A.V. Lebedev, K.I. Morozov. Effect of interaction between particles on the magnetostatic properties of magnetic liquids. Magnetohydrodynamics, vol. 23 (1987), no.1, pp. 31-36.

[3] H. Mamiya, I. Nakatani, T. Furubayashi. Phase transitions of ironnitride magnetic fluids. Phys. Rev. Lett., vol. 84 (2000), no. 26, pp. 6106-6109.

[4] A.A. Minakov, I.A. Zaitsev, U.I. Lesnih. Critical behaviour of magnetic fluids near superparamagnetic-dipole-glass transition. J. Magn. Magn. Mater., vol. 85 (1990), no. 1-3, pp. 60-62.

[5] K. OGrady, J. Popplewell, S.W. Charles. Initial susceptibility of ferrofluids. J. Magn. Magn. Mater., vol. 39 (1983), no. 1-2, pp. 56-58.

[6] Y.I. Dikanskit, A.G. Ispiryan, S.A. Kunikin, A.V.Radionov. On the nature of the maximum in the temperature dependence of magnetic liquid susceptibility. Tech. Phys., vol. 60 (2015), no. 8, pp. 1204-1207.

[7] E. Blums, A. Cebers, M. Maiorov. Magnetic Fluids (De Gruyter, 1997).

[8] J.Zhang, C. Boyd, W. Luo. Two mechanisms and a scaling relation for dynamics in ferrofluids. Phys. Rev. Lett., vol. 77 (1996), no. 2, pp. 390-393.

Received 27.12.2017 